\title{
Reto diagnóstico y terapéutico en la hemorragia intracerebral por aneurisma de la arteria lenticuloestriada distal
}

\section{Diagnostic and therapeutic challenge in intracerebral hemorrhage due to aneurysm of distal lenticulostriate artery}

\author{
José A. Figueroa-Sánchez', Enrique Caro-Osorio ${ }^{1}$, Héctor R. Martínez-Rodríguez² y \\ José C. Herrera-Castro ${ }^{1 *}$
}

${ }^{1}$ Servicio de Neurocirugía; ${ }^{2}$ Servicio de Neurología. Centro Médico Zambrano Hellion, Tecnológico de Monterrey, Escuela de Medicina y Ciencias de la Salud, Monterrey, Nuevo León, México

\section{Resumen}

Objetivo: Presentar un caso atípico de hemorragia intracerebral profunda causada por la ruptura de un aneurisma de la arteria lenticuloestriada distal; se revisa la bibliografía y se analizan la fisiopatología y el tratamiento. Se presentó a la sala de emergencias una mujer de 42 años con hemiparesia leve del hemicuerpo derecho. Se la diagnosticó con una hemorragia aguda en los núcleos de la base izquierdos. La panangiografía cerebral reveló un microaneurisma de la porción distal de la arteria lenticuloestriada izquierda. Se recomendó tratamiento conservador bajo estrecha vigilancia neurológica. Después de unos cuantos días de hospitalización, los síntomas neurológicos de la paciente mejoraron y la paciente recibió el alta. La paciente ha permanecido asintomática durante más de seis meses. Los aneurismas de la arteria lenticuloestriada distal son extremadamente raros.

Palabras Clave: Aneurisma. Hemorragia cerebral. Arteria lenticuloestriada.

\begin{abstract}
Objective: To present an atypical case of deep intracerebral hemorrhage caused by the rupture of an aneurysm of the distal lenticulostriate artery. A 42-year-old woman presenting with right-sided mild hemiparesis was diagnosed with an acute left-sided hemorrhage within the basal nuclei. Cerebral angiography revealed a microaneurysm of the distal portion of the left lenticulostriate artery. Conservative treatment under close neurological surveillance was recommended. After a few days of hospital stay, the patient's neurological symptoms improved, and she was discharged. The patient has remained asymptomatic for more than 6 months. Aneurysms of the distal lenticulostriate artery are extremely rare.
\end{abstract}

Key Words: Aneurysm. Cerebral hemorrhage. Lenticulostriate artery.

\section{Correspondencia:}

*José C. Herrera-Castro

Avda. Batallón de San Patricio, 112

Col. Real San Agustín

Fecha de recepción: 14-01-2019

C.P. 66278 , San Pedro Garza García, N.L., México

E-mail: jcherrera.c4@ gmail.com 0009-7411/@ 2019 Academia Mexicana de Cirugía. Publicado por Permanyer. Este es un artículo open access bajo la licencia CC BY-NC-ND (http://creativecommons.org/licenses/by-nc-nd/4.0/).

Cir Cir. 2020;88(3):366-369 Contents available at PubMed www.cirugiaycirujanos.com 


\section{Antecedentes}

Los accidentes cerebrovasculares hemorrágicos representan 10 a $20 \%$ de todos los eventos vasculares cerebrales $(E V C)^{1-3}$. La hemorragia intracerebral (HIC) profunda en los núcleos de la base es común y puede desarrollarse debido a múltiples causas, las más de las veces relacionada con hipertensión arterial sistémica (HAS). La ruptura de un aneurisma de la arteria lenticuloestriada (ALE) es un suceso en extremo raro y más aún cuando se presenta en la porción distal del vaso ${ }^{4}$. La angiografía cerebral es el estudio más sensible para detectar aneurismas en la ALE; sin embargo, casi nunca se realiza en pacientes con HIC profunda ${ }^{2}$. En la actualidad no hay un consenso sobre el tratamiento de esta afección debido a la baja incidencia de ruptura de aneurismas en la ALE como causa de una HIC profunda sin relación con HAS. En este documento se informa un caso de ruptura de aneurisma de ALE distal que se trató de manera conservadora.

\section{Caso clínico}

Mujer de 42 años, previamente sana, que se presenta a la sala de emergencias con hemiparesia derecha ( $4 / 5$ de la escala de Lovett), sutil parálisis facial central derecha, sin afasia, con una escala de EVC del NIHSS (National Institute of Health Stroke Scale) de 4 y puntuación de la clasificación modificada de 2 . A su llegada se activó el equipo de atención de EVC. La tomografía axial computarizada (TC) de cráneo reveló una hemorragia en los núcleos de la base izquierdos (Fig. 1A,B). La paciente estaba alerta y hemodinámicamente estable. Ingresó a la unidad de cuidados intensivos para su tratamiento médico y se realizó una resonancia magnética (RM) cerebral con contraste. (Fig. 1C,D).

Las pruebas de laboratorio fueron normales o negativas, incluido el recuento de plaquetas, perfil de coagulación, perfil de autoinmunidad (anticuerpos antinucleares, ANCA, proteína $C$ reactiva y complemento C3-C4-C50), proteína C, proteína S, antitrombina III y factor de Leiden V. El análisis volumétrico del hematoma fue de $8 \mathrm{~cm}^{3}$. Después de descartar las principales causas de HIC, se solicitó una angiografía cerebral tres días después del ingreso, en la que se encontró un pequeño aneurisma en la porción distal de la arteria lenticuloestriada izquierda. Se descartó la posibilidad de un aneurisma de causa infecciosa
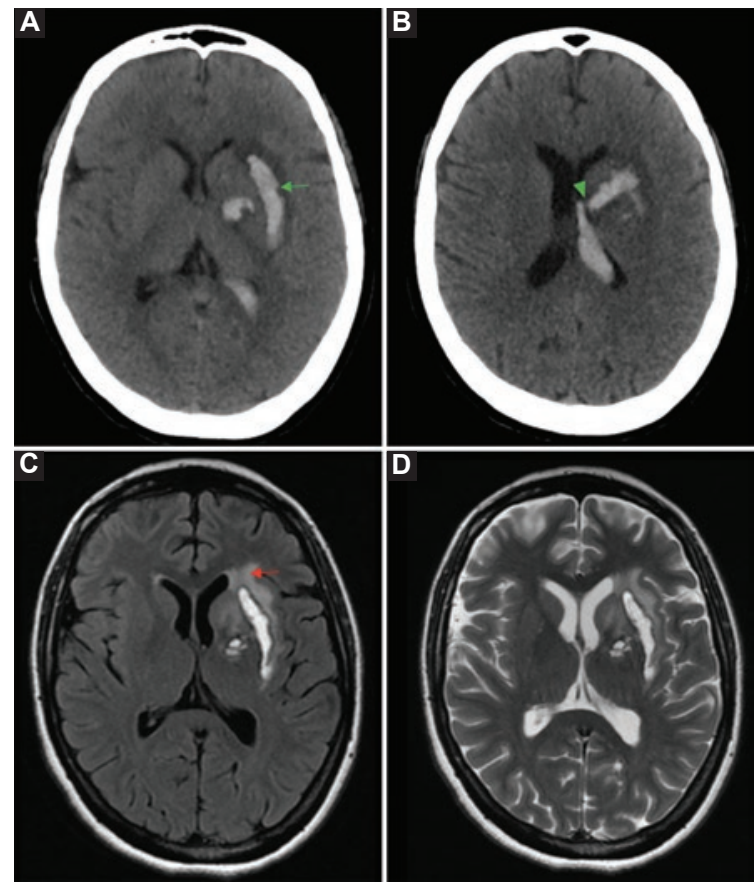

Figura 1. Corte axial en TC craneal que muestra una HIC profunda de $8 \mathrm{ml}$ y que compromete sobre todo el putamen izquierdo (flecha verde). A: Extensión intraventricular (punta de flecha). B: RM cerebral: secuencia T1 de corte axial. C: Hemorragia de los núcleos de la base izquierdos con edema perilesional (flecha roja). D: La imagen de RM axial en secuencia T2 no identificó aneurisma o malformación vascular.

debido a la ausencia de factores de riesgo, clínica inespecífica y estudios de laboratorio negativos para infección y por lo que no se instituyó antibioticoterapia. Se consideró una embolización endovascular supraselectiva para el aneurisma, si bien la anatomía vascular de la porción proximal de la ALE se encontró estrecha y tortuosa, por lo que se descartó este enfoque terapéutico al considerarse de alto riesgo (Figs. 2 y 3).

Se realizó una prueba funcional de Wada que demostró predominio del hemisferio cerebral izquierdo. Un equipo multidisciplinario analizó el caso y, tras tomar en consideración el deseo de la paciente, se decidió un enfoque de tratamiento conservador.

Siete días después de la admisión, la paciente recibió el alta con una ligera hemiparesia del lado derecho y sin parálisis facial. En el seguimiento clínico a los seis y 12 meses, la paciente se encontró asintomática (NIHSS 0, mRS 0).

El tratamiento conservador en esta paciente se basó en el tipo y tamaño del aneurisma, la ubicación profunda y la elocuencia y, aunque permanece asintomática después de un año del episodio, se sugirieron estudios de neuroimagen de seguimiento que la paciente rechazó. 

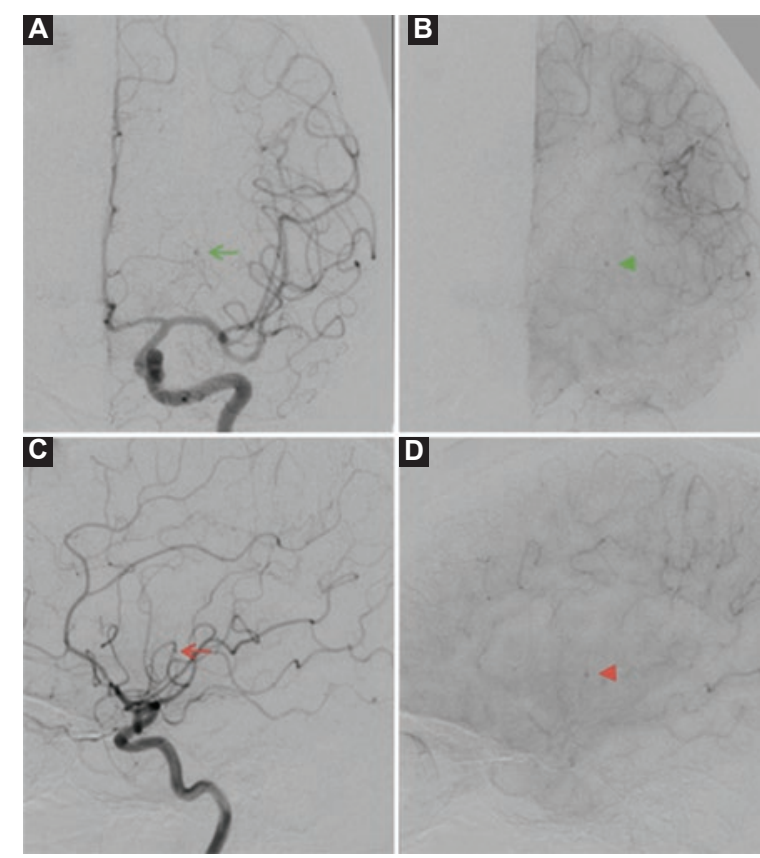

Figura 2. Angiografía cerebral. Fase arterial temprana de la arteria carótida interna izquierda. A: Proyección AP que muestra una sutil dilatación anormal de la ALE distal (flecha verde), que corresponde a un microaneurisma sacular de $1.4 \times 1.5 \times 1.5 \mathrm{~mm}$, con un volumen aproximado de $0.15 \mathrm{~cm}^{3}$. B: Proyección AP en fase arterial tardía; el material de contraste permanece dentro del aneurisma (punta de flecha verde). Fase arterial temprana. C: Vista lateral en la que se observa sutilmente una dilatación anormal de la ALE distal (flecha roja). Fase arterial tardía. D: Vista lateral; el contraste permanece dentro del aneurisma después de la fase arterial temprana (punta de flecha roja).

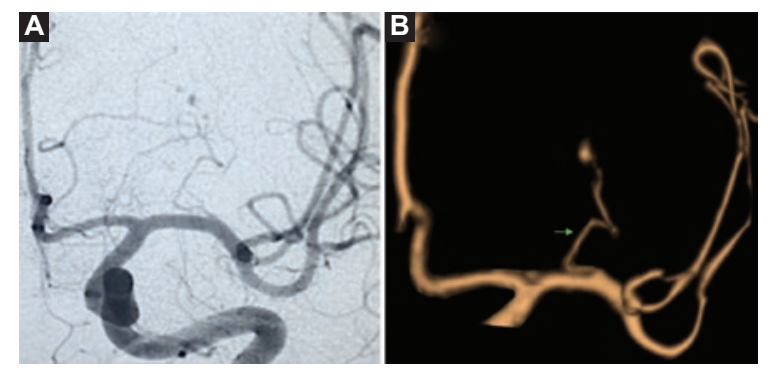

Figura 3. Angiografía cerebral. A: fase arterial temprana del angiograma de la carótida izquierda con un primer plano en la ALE. B: reconstrucción tridimensional que muestra un aspecto estrecho y tortuoso de la angioconfiguración de la porción proximal de la ALE.

\section{Discusión}

En pacientes con HIC espontánea, el tratamiento quirúrgico y otros tratamientos invasivos son aún controversiales. No obstante, las recomendaciones en guías actuales no incluyen a pacientes con lesiones estructurales causantes del $\mathrm{HIC}$ como los aneurismas cerebrales ${ }^{2}$.

En la fisiopatología de los HIC profundos se han descrito los microaneurismas en pacientes con HAS, que por lo general afectan a las arterias perforantes (aneurismas de Charcot-Bouchard), de los cuales el $80 \%$ se ha observado en la ALE; sin embargo, hay poca evidencia de microaneurismas en la ALE de pacientes jóvenes sin $\mathrm{HTA}^{5-7}$.

Vargas, et al. ${ }^{8}$ realizaron la descripción más completa de aneurismas en la ALE, presentaron una serie de 48 casos de aneurismas en la ALE y los clasificaron en tres tipos. El tipo 1 incluye al aneurisma que surge de la arteria cerebral media (ACM) junto al sitio de nacimiento de la ALE; en el tipo 2, el aneurisma se origina en la porción proximal de la ALE; y el tipo 3 comprende los aneurismas distales de la ALE. Con base en esta clasificación, se estableció que este caso es de tipo $3^{8}$.

Los aneurismas de la ALE se han relacionado con varias enfermedades, como la HAS, la enfermedad de Moya-Moya y el lupus eritematoso sistémico ${ }^{8,9}$. En el abordaje diagnóstico habitual de un HIC profundo, en pacientes en quienes usualmente no se realiza angiografía cerebral, la angiotomografía ha demostrado ser sensible en la detección de pequeños aneurismas; no obstante, algunas lesiones pueden no observarse. Tan, et al. ${ }^{10}$ demostraron el "signo de spot" en la TAC con contraste (foco de realce de contraste dentro del hematoma) de un paciente con un HIC de núcleos de la base causado por un aneurisma en la ALE.

En pacientes jóvenes con HIC profundo, en los que se descartaron otras causas de sangrado, es importante confirmar este tipo de aneurismas distales en la ALE. Pese a ello, un pequeño aneurisma en la ALE distal puede pasar inadvertido, incluso en la angiografía cerebral ${ }^{11}$.

Debido al reducido número de casos informados en las publicaciones médicas, no existe un consenso acerca de la mejor forma de tratamiento de los aneurismas de la ALE. Algunos autores han intentado usar endoprótesis con resultados muy disímiles. Otras opciones como la radiocirugía ${ }^{12-14} \mathrm{O}$ la terapia endovascular $^{8}$ también se han descrito, pero sin pruebas suficientes para generalizar este tipo de tratamientos. Se requieren estudios prospectivos y multicéntricos para analizar a un mayor número de pacientes, generalizar resultados y emitir recomendaciones diagnósticas y terapéuticas.

\section{Conflicto de intereses}

Los autores declaran no tener ningún conflicto de intereses. 


\section{Responsabilidades éticas}

Protección de personas y animales. Los autores declaran que para esta investigación no se han realizado experimentos en seres humanos ni en animales.

Confidencialidad de los datos. Los autores declaran que han seguido los protocolos de su centro de trabajo sobre la publicación de datos de pacientes.

Derecho a la privacidad y consentimiento informado. Los autores han obtenido el consentimiento informado de los pacientes o sujetos referidos en el artículo. Este documento obra en poder del autor de correspondencia.

\section{Bibliografía}

1. An SJ, Kim TJ, Yoon BW. Epidemiology, risk factors, and clinical features of intracerebral hemorrhage: an update. J Stroke. 2017;19:3.

2. Hemphill JC, Greenberg, SM, Anderson CS, Becker K, Bendok BR, Cushman MPA. Guidelines for the management of spontaneous intracerebral hemorrhage: a guideline for healthcare professionals from the American Heart Association/American Stroke Association. Stroke 2015;46(7):2032-2060.
3. Feigin VL, Lawes CM, Bennett DA, Barker-Collo SL, Parag V. Worldwide stroke incidence and early case fatality reported in 56 population-based studies: a systematic review. Lancet Neurol 2009;8:355-69.

4. Choo YS, Kim YB, Shin YS, Joo JY. Deep intracerebral hemorrhage caused by rupture of distal lenticulostriate artery aneurysm: a report of two cases and a literature review. J Korean Neurosurg Soc 2015;58: 471-75.

5. Amin OS. Simultaneous hypertensive intracerebral haemorrhages: what are the odds? BMJ case reports. 2013;bcr2012008047.

6. Muhammed AOS, Zangana HM. Recurrent hypertensive intracerebral hemorrhage: a case series from a single institution in Iraq. Gaziantep Medical Journal. 2012;18(3):169-172.

7. Kumar Abbas $F$ (eds). Robbins and Cotran pathologic basis of disease. $7^{\text {th }}$ ed. China: Elsevier, 2005

8. Vargas J, Walsh K, Turner R, Chaudry I, Turk A, Spiotta A. Lenticulostriate aneurysms: a case series and review of the literature. J Neurointerv Surg. 2015;7:194-201.

9. Kidoguchi J, Chiba M, Murakami T. A case of systemic lupus erythematosus associated with an aneurysm of the lenticulostriate artery. No Shinkei Geka. 1987:15:1221-5.

10. Tan LA, Kasliwal MK, Johnson AK, Lopes DK. The "spot sign" secondary to a ruptured lenticulostriate artery aneurysm. Clin Imaging. 2014;38:508-9.

11. Sakai K, Mizumatsi S, Terasaka K, Sugatani H, Higashi T. Surgical treatment of a lenticulostriate artery aneurysm. Neurol Med Chir. 2005; 45:574-77.

12. Horn EM, Zabramski JM, Feiz-Erfan I, Lanzino G, McDougall CG. Distal lenticulostriate artery aneurysm rupture presenting as intraparenchymal hemorrhage: case report. Neurosurg. 2004;55:E708-12.

13. Narayan P, Workman MJ, Barrow DL. Surgical treatment of a lenticulostriate artery aneurysm: case report. J Neurosurg. 2004; 100:340-2.

14. Lan Z, Li J, You C, Chen, J. Successful use of gamma knife surgery in a distal lenticulostriate artery aneurysm intervention. Br J Neurosurg. 2012; 26:89-90. 